\title{
Enhanced droplet spreading due to thermal fluctuations
}

\author{
A M Willis ${ }^{1,2}$ and J B Freund ${ }^{1,3}$ \\ ${ }^{1}$ Department of Mechanical Science and Engineering, College of Engineering, \\ University of Illinois at Urbana-Champaign, 1206 West Green Street, Urbana, IL 61801, USA \\ ${ }^{2}$ College of Medicine at Urbana-Champaign, University of Illinois at Urbana-Champaign, \\ USA \\ ${ }^{3}$ Department of Aerospace Engineering, University of Illinois at Urbana-Champaign, USA \\ E-mail: jbfreund@illinois.edu
}

Received 3 June 2009, in final form 3 August 2009

Published 29 October 2009

Online at stacks.iop.org/JPhysCM/21/464128

\begin{abstract}
The lubrication equation that governs the dynamics of thin liquid films can be augmented to account for stochastic stresses associated with the thermal fluctuations of the fluid. It has been suggested that under certain conditions the spreading rate of a liquid drop on a surface will be increased by these stochastic stresses. Here, an atomistic simulation of a spreading drop is designed to examine such a regime and provide a quantitative assessment of the stochastic lubrication equation for spreading. It is found that the atomistic drop does indeed spread faster than the standard lubrication equations would suggest and that the stochastic lubrication equation of Grün et al (2006 J. Stat. Phys. 122 1261-91) predicts the spread rate.
\end{abstract}

(Some figures in this article are in colour only in the electronic version)

\section{Background}

The dynamics of thin liquid films are important in a range of applications, and in most cases thin liquid film predictions can be made via simplifications of the full Navier-Stokes equations. This simplification is usually founded on an assumption of a small capillary number $C a \equiv \eta U / \gamma$, where $U$ is the relevant velocity scale, $\eta$ is the liquid viscosity, and $\gamma$ is the free surface tension. For our discussions $\eta$ and $\gamma$ are assumed constant. When the thickness of the film $d$ is much smaller than its other spatial dimensions, say $\lambda$, a long-wave approximation $\lambda \gg d$ is also employed giving the so-called lubrication limit of the flow equations. With these assumptions and standard no-slip and stress-balance boundary conditions at the wall and free surface, a single partial differential equation can be constructed that governs the film thickness $h(x, t)$ [12]. Further modifications have been employed to model the behavior of evaporating films [2].

However, experimental observations [11] and molecular simulations [16] suggest that thermal stresses, which are not included in the standard analysis, can affect the fluid film mechanics. Moseler and Landman [16] demonstrated the necessity of including thermal stresses to even qualitatively reproduce the evolving shape of the surface tension instability of a nanometer-scale jet. Hennequin et al [11] suggest in regard to a colloid-polymer model that the amplitude of thermal fluctuations per se will affect the size distribution in droplet formation.

To account for such effects associated with thermal fluctuations, Grün et al [7] incorporated stochastic stresses into the lubrication model and suggested that thermal fluctuations will decrease the rupture time for unstable thin liquid films. Starting from a similar formulation, Davidovitch et al [4] predicted that the spreading of a self-similar droplet on a smooth, flat surface can be primarily driven by these fluctuating thermal stresses, which will increase the power-law spreading rate from the standard surface tension spreading Tanner's law [20] of $t^{1 / 7}$ up to $t^{1 / 4}$. This predicted enhanced spreading rate is the focus of this paper. We are not able to study this specific self-similar regime, but we are able to directly compare predictions of atomistic simulation with the stochastic lubrication formulation of Grün et al [7].

Thermal fluctuations in thin liquid films are primarily manifested in perturbations of the fluid interface. The scale of these perturbations are characterized by a thermal length $l_{\mathrm{T}}=\sqrt{k_{\mathrm{B}} T / \gamma}$, with $k_{\mathrm{B}} T$ the thermal energy scale of the fluid. For normal fluids this length scale is, of course, of atomic dimensions, with concomitant timescales significantly smaller than microseconds. Because of the difficulty in measuring fluid flow at such small length and short timescales, direct 
examination of the temporal evolution of such fluid flows is limited. Most experimental observations are based upon one of two models: colloid-polymer interfaces $[1,11]$ or polystyrene thin films $[13,14,24,19]$. Because of their effectively low surface tensions and high viscosities, the interfaces of colloidpolymer films have significantly larger perturbation length and timescales than their simple-fluid counterparts. However, the complex and poorly characterized fluid properties of colloidpolymers hinder direct comparison to continuum fluid models. Polystyrene films are advantageous in that they can be formed to precise thicknesses and have high enough viscosities to slow timescales, but it is difficult to sort out any thermal stress phenomenology from behavior associated with their viscoelastic character [13, 24], local variations in viscosity caused by confinement [14, 23], and non-local interactions with the wall [19]. These complexities also hinder direct comparison to continuum fluid models.

Atomistic simulations of model fluids have been used effectively to investigate the role of stochastic thermal stresses [16], and we take this approach here to assess the stochastic lubrication equation for spreading. If we accept that the model provided by empirical atomic interaction potentials is sufficiently realistic to test the assertions in the stochastic lubrication equations, then the primary limitation of the scope of such an investigation is its computational cost. The key distinction between this work and previous atomistic simulations of spreading drops $[10,8]$ is that our simulation is specifically designed to examine the thermally dominated regime by using a large, three-dimensional, relatively longtime simulation of a simple-fluid low viscosity drop.

\section{Theoretical framework}

\subsection{Thermal stresses}

A thermally augmented lubrication equation [7] can be developed by adding a term $\nabla \cdot \mathcal{S}$ into the Navier-Stokes momentum equation that models the stochastic stresses that arise via the thermal fluctuations:

$$
\rho \frac{D \mathbf{u}}{D t}=-\nabla p+\eta \nabla^{2} \mathbf{u}+\nabla \cdot \mathcal{S}
$$

where $\mathbf{u}$ is the fluid velocity, $p$ is the pressure, and $\mathcal{S}$ is a Gaussian white noise stochastic stress tensor. This stress has zero mean $(\langle\mathcal{S}\rangle=0)$ and its autocorrelation

$$
\left\langle\mathcal{S}_{i j}(\mathbf{r}, t) \mathcal{S}_{k l}\left(\mathbf{r}^{\prime}, t^{\prime}\right)\right\rangle=2 \eta k_{\mathrm{B}} T \delta\left(\mathbf{r}-\mathbf{r}^{\prime}\right) \delta\left(t-t^{\prime}\right)\left(\delta_{i l} \delta_{j m}+\delta_{i m} \delta_{j l}\right)
$$

is fixed via the fluctuation-dissipation theorem.

Including this stochastic stress in their development of thin-film equations, Grün et al [7] showed that it modifies the governing equation for film thickness $y=h(x, t)$, where $x$ is the coordinate parallel to the wall in the direction of spreading. To develop such a one-dimensional model requires homogenization in $z$, parallel to the contact line, which is tantamount to averaging over a length $W$ in the $z$ direction. We will see that our computational domain is designed to be a thin three-dimensional slab of width $W$ in $z$, which is both computationally convenient and precisely sets $W$. The resulting governing equation is

$$
\frac{\partial h}{\partial t}=\frac{\partial}{\partial x}\left[-\frac{\gamma}{3 \eta} h^{3} \frac{\partial^{3} h}{\partial x^{3}}+\beta h^{3 / 2} \mathcal{N}(x, t)\right],
$$

where $\beta \equiv \sqrt{2 k_{\mathrm{B}} T / 3 \eta W}$ and $\mathcal{N}(x, t)$ is a normalized Gaussian white noise random variable, with $\langle\mathcal{N}(x, t)\rangle=0$ and

$$
\left\langle\mathcal{N}(x, t) \mathcal{N}\left(x^{\prime}, t^{\prime}\right)\right\rangle=\delta\left(x-x^{\prime}\right) \delta\left(t-t^{\prime}\right) .
$$

Full details of this derivation are provided by Grün et al [7].

\subsection{Evaporation}

A low molecular weight polymer is used for these simulations. This has the advantage of having a relatively low liquid-state viscosity, which increases the rate of spreading and thereby increases the scope of this study, but it also has a drawback of non-negligible evaporation, which we therefore include in our thin-film model. The model we employ uses a linearized non-equilibrium evaporation model of Scrage [18], which has been used to study the dynamics of small confined bubbles [2] as well as an atomistic meniscus [6]. In this model, the mass flux of evaporation $J^{*}$ is linearly proportional to the pressure jump across the interface,

$$
J^{*}=\rho_{\mathrm{v}}\left(\frac{2 \pi}{\mathcal{R} T^{\mathrm{i}}}\right)^{1 / 2}\left(\frac{p_{\mathrm{v}}^{\mathrm{e}}}{p_{\mathrm{v}}}-1\right),
$$

with $\rho_{\mathrm{v}}$ the vapor density, $\mathcal{R}$ the mass-specific gas constant, $T^{\mathrm{i}}$ the temperature at the fluid-vapor interface, and $p_{\mathrm{v}}^{\mathrm{e}}$ and $p_{\mathrm{v}}$ the equilibrium vapor pressure and local pressure at the interface, respectively.

For non-dimensionalization, we follow Ajaev and Homsy [2] and define velocity and pressure scales

$$
U=\frac{k T_{\mathrm{s}}^{*}}{\rho \mathcal{L} l} \quad p=\frac{\gamma}{l}
$$

with $k$ the thermal conductivity of the fluid, $\mathcal{L}$ the latent heat of vaporization, $T_{\mathrm{s}}^{*}$ the equilibrium saturation temperature, $\rho$ the liquid density, and $l$ an appropriate macroscopic length scale, which we take to be the $x$-domain length of the simulation. With these, the non-dimensional mass flux is

$$
J=\frac{\delta_{\mathrm{v}}\left(p-p_{\mathrm{v}}\right)+T^{\mathrm{i}}-1}{K},
$$

where

$$
K=\frac{\rho U \sqrt{2 \pi \mathcal{R} T_{\mathrm{s}}^{*}}}{2 \rho_{\mathrm{v}} \mathcal{L}} \quad \text { and } \quad \delta_{\mathrm{v}}=\frac{\gamma}{\rho \mathcal{L} l} .
$$

As explained by Ajaev et al [2], $K$ represents the relative importance of kinetic effects in changing local vapor pressure and $\delta_{\mathrm{v}}$ relates the change in local pressure of the liquid to changes in local evaporation temperature. 


\subsection{Capillary scaling}

To balance the effects of pressure driven flow, evaporation, and thermally driven flow, we applied the scalings of Ajaev and Homsy [2],

$$
\begin{gathered}
x=C a^{1 / 6} l \hat{x}, \quad y=C a^{1 / 3} l \hat{y}, \quad h=C a^{1 / 3} l \hat{h}, \\
t=C a^{2 / 3} \frac{l}{U} \hat{t}, \quad u=C a^{-1 / 2} U \hat{u}, \quad v=C a^{-1 / 3} U \hat{v}, \\
J=C a^{-1 / 3} \hat{J}, \quad K=C a^{1 / 3} \hat{K} .
\end{gathered}
$$

To retain the stochastic stresses in the $\mathrm{Ca} \rightarrow 0$ limit we similarly choose

$$
\beta=C a^{-1 / 4} \hat{\beta} .
$$

Following the straightforward steps in Ajaev and Homsy [2], the resulting lubrication equation is

$$
\frac{\partial \hat{h}}{\partial \hat{t}}=\frac{\partial}{\partial \hat{x}}\left[-\hat{h}^{3} \frac{\partial^{3} \hat{h}}{\partial \hat{x}^{3}}+\hat{\beta} \hat{h}^{3 / 2} \mathcal{N}(\hat{x}, \hat{t})\right]+\frac{\delta_{\mathrm{v}} \frac{\partial^{2} \hat{h}}{\partial \hat{x}^{2}}}{\hat{K}+\hat{h}},
$$

where the terms on the right-hand side correspond respectively to pressure, thermal fluctuations, and evaporation of the film. Equation (10) is simplified by several assumptions, the first being that the saturation temperature is equivalent to the wall temperature. This assumption is justified given that our simulation domain was essentially isothermal, with the temperature maintained by using a weak stochastic thermostat on the solid wall. Upon inspection, there existed no measurable thermal gradients within the domain. This is consistent with the fact the $\hat{K}$ is approximately 400 times greater than $\hat{h}$ for the precursor film, which essentially minimizes thermal gradients for the majority of the surface area of the fluid interface. A second assumption is that there is no disjoining pressure contribution from van der Waals or any other forces. This assumption will be discussed in section 3.1.

Another key point that must be addressed is that (10) does not formally obey the fluctuation-dissipation theorem. The fluctuation-dissipation theorem relates the energy of fluctuations of a system to the energy dissipation of an infinitesimal external perturbation of the system, yielding in the equations of motion a fluctuation term with a magnitude proportional to the square root of the linearized dissipative term. However (10) only contains the fluctuations from the interaction with viscous dissipation, without accounting for the fluctuations associated with the evaporative dissipation. To justify this we linearize the deterministic dimensional version of (10) for a single wavenumber component $q$ by making the substitution

$$
h(x, t)=H+\delta h(t) \cos (q x) .
$$

Assuming that $H \gg \delta h(t)$, leads to

$$
\begin{aligned}
& \frac{\partial \delta h(t)}{\partial t}=(\underbrace{\frac{H^{3} \gamma q^{4}}{\eta}}_{\text {Viscous }}-\underbrace{\frac{2 k T_{\mathrm{s}} \rho_{\mathrm{v}} \gamma \mathcal{R} q^{2}}{\rho^{2} \mathcal{L}\left(k \sqrt{2 \pi} T_{\mathrm{s}}^{3 / 2}+2 H \mathcal{L} \mathcal{R} \rho_{\mathrm{v}}\right)}}_{\text {Evaporative }}) \\
& \times \delta h(t),
\end{aligned}
$$

and we compare the relative importance of the viscous versus evaporative dissipation terms by substituting values of $q$ and $H$ corresponding to the length and thickness of the drop. This long-wavelength choice for $q$ maximizes the relative importance of the evaporation dissipation. Substituting these values and the physical properties of the liquid into the two linear dissipation terms of (12) suggests the viscous dissipation term is $1.8 \times 10^{6}$ larger than the evaporative dissipation. Thus because the magnitude of the fluctuations in this linear model is proportional to the square root of the dissipation term, the viscous fluctuation is expected to be over 1000 times greater than the evaporative fluctuation term, justifying its neglect in the current study. Although the fluctuating evaporation is negligible, evaporation itself is still important due local variations in pressure because of the interface curvature, and this is accounted for in the evaporation model.

\section{Methods}

\subsection{Atomistic simulations}

All atoms in the simulation are modeled with a LennardJones pair potential, which includes a $1 / r^{6}$ dispersion (van der Waals) attraction and an empirical $1 / r^{12}$ repulsion:

$$
u_{\mathrm{LJ}}(r)= \begin{cases}4 \varepsilon\left[\frac{\sigma^{6}}{r^{6}}-\frac{\sigma^{12}}{r^{12}}\right] & \text { for } r \leqslant r_{\mathrm{c}} \\ 0 & \text { for } r>r_{\mathrm{c}}\end{cases}
$$

where $\varepsilon$ is the interaction energy, $r$ is the distance between the two atoms, and $\sigma$ is the interaction length scale. The simulations employ a cutoff radius $r_{\mathrm{c}}=2.5 \sigma$. Most physical properties are insensitive to this $r_{\mathrm{c}}$, but it does decrease the effective surface tension by a factor of about two [21]. This is accounted for in the continuum model as discussed in section 3.2. Because the drop and the precursor film are thicker than this $2.5 \sigma$ cutoff, there is also in effect no disjoining pressure, which is why it was not included in the analysis leading to (10). This is advantageous in that it simplifies the comparison between the molecular dynamics and solutions of (10). Disjoining pressure is not expected to significantly alter the spreading of a drop of the kind considered [4].

To suppress evaporation and condensation, which would muddle results, Lennard-Jones atoms are joined into polymers using the finite extensible nonlinear elastic (FENE) potential $[15,9]$,

$$
u_{\mathrm{f}}= \begin{cases}-\frac{1}{2} k R_{o}^{2} \log \left[1-\frac{r^{2}}{R_{o}^{2}}\right] & \text { for } x<R_{o} \\ \infty & \text { for } r \geqslant R_{o}\end{cases}
$$

where $k$ is the spring strength and $R_{o}$ is the reference length of the polymer. Taking $k=30 \epsilon / \sigma^{2}$ and $R_{o}=1.5 \sigma$ has been shown to provide realistic packing without imposing any significant new restriction on the numerical time step [15].

The atomistic simulation contained 68134 fluid atoms and 44200 wall atoms. The fluid atoms were in two-atom 'polymers'. Each atom had a mass of $m_{\mathrm{f}}=m$. The interaction 


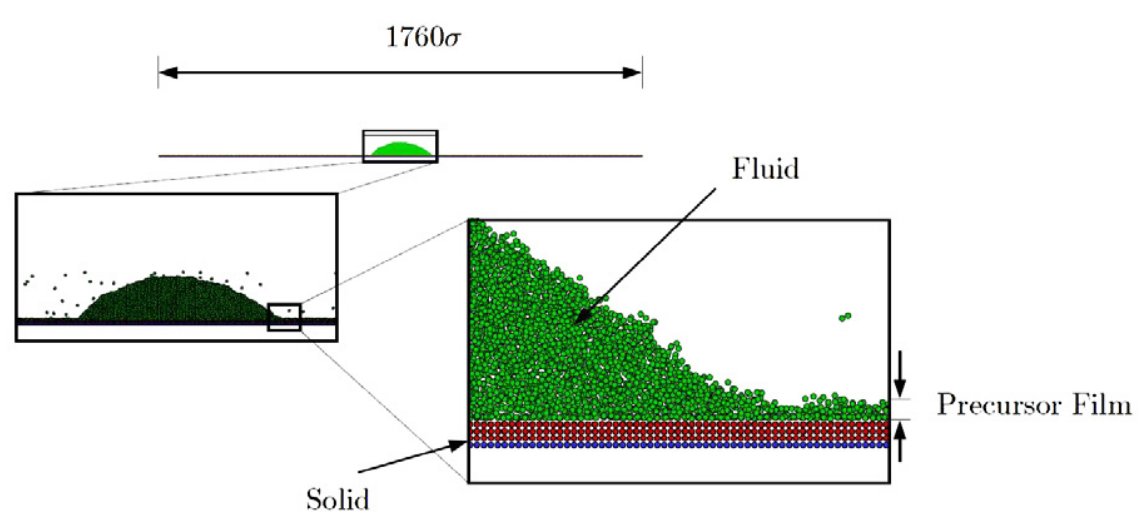

Figure 1. Simulation domain.

Table 1. Atomistic parameters.

\begin{tabular}{lll}
\hline Symbol & Description & Value \\
\hline$m$ & Atom mass & $1.3277 \times 10^{-26} \mathrm{~kg}$ \\
$\epsilon / k_{\mathrm{B}}$ & Lennard-Jones interaction energy & $118 \mathrm{~K}$ \\
$\sigma$ & Lennard-Jones length scale & $3.405 \times 10^{-10} \mathrm{~m}$ \\
\hline
\end{tabular}

energy between all fluid and solid atoms was the same: $\varepsilon_{\mathrm{ff}}=$ $\varepsilon_{\text {sf }}=\varepsilon$. The values of the molecular parameters were set to match that of liquid argon as listed in table 1. As shown in figure 1, the solid atoms were arranged into 4 layers of an FCC lattice of length $1760 \sigma$ in $x$ and depth (into the page) of $8 \sigma$ in $z$. The melting temperature of the solid atoms was decreased by taking $m_{\mathrm{s}}=10 \mathrm{~m}$ and $\varepsilon_{\mathrm{ss}}=10 \varepsilon$.

Time integration was via the velocity Verlet algorithm, which has excellent but not exact conservation properties. Because of the long times of these simulation, a weak thermostat was applied to maintain the temperature. Using a weak stochastic Andersen [3] thermostat, solid substrate atoms were randomly chosen and then reassigned a velocity from the appropriate Boltzmann distribution. Because the thermostat was weak and only modified the velocity of the solid atoms, spreading dynamics should not be affected by this procedure.

The initial condition was generated by 'cutting' out a droplet shape from a flat thermally equilibrated thick fluid film. A precursor film was also included over the whole domain (see figure 1), whose thickness was set to be slightly larger than the cutoff radius of the potential (13). This choice simplifies analysis by essentially removing any disjoining pressure effects. Because our cutting procedure introduces a non-equilibrated shape to our drop from an equilibrated flat profile, there is an initial period of local equilibration where a large amount of evaporation takes place. This portion of the simulation was not included in results and our 'time zero', from which the initial conditions for (10) were defined, was a time when this initial period of equilibration seemed to have ceased. Different choices for this 'time zero' do not alter the conclusions of this work.

\subsection{Lubrication equation solver}

Equation (10) was solved with an implicit Crank-Nicholsonlike time integration and a Fourier collocation discretization in
Table 2. Properties of the molecular fluid.

\begin{tabular}{lll}
\hline Symbol & Description & Value \\
\hline$T$ & Equilibrium temperature & $100 \mathrm{~K}$ \\
$\rho$ & Fluid density & $1365 \mathrm{~kg} \mathrm{~m}^{-3}$ \\
$\rho_{\mathrm{v}}$ & Vapor density & $33.6 \mathrm{~kg} \mathrm{~m}^{-3}$ \\
$\mathcal{L}$ & Latent heat of vaporization & $649 \mathrm{~kJ} \mathrm{~kg}^{-1}$ \\
$k$ & Thermal conductivity & $0.0991 \mathrm{~J} \mathrm{~m}^{-1}$ \\
$\eta$ & Viscosity & $1.101 \times 10^{-4} \mathrm{~kg} \mathrm{~ms}^{-1}$ \\
$\gamma$ & Surface tension & $1.07 \times 10^{-2} \mathrm{~kg} \mathrm{~m}^{2} \mathrm{~s}^{-2}$ \\
\hline
\end{tabular}

$x$. The implicit solve was by Newton's method. The stochastic noise term was generated every time step and included as a forcing term. The initial condition was taken as the measured interface of the molecular spreading drop as defined by the density isopleth $\rho_{\mathrm{c}}=\rho_{\mathrm{f}} / 10$, where $\rho_{\mathrm{f}}$ is the bulk fluid density. Results are insensitive to our choice of $\rho_{\mathrm{c}}$. Ten such stochastic solutions are averaged to produce ensemble averaged spreading solutions.

For direct comparison between the molecular simulation and the predictions of the continuum model (10), the physical parameters of the molecular fluid needed to be independently determined (see table 2). Using small samples of the molecular fluid, thermal conductivity $k$, viscosity $\eta$, and surface tension $\gamma$ were all calculated using established techniques $[17,5]$. The latent heat of vaporization $\mathcal{L}$, which is important for modeling evaporation, was less straightforward to estimate. To do this the critical temperature and pressure of the fluid were measured by plotting the equilibrium fluid film thickness in a closed container as a function of temperature. This plot established the critical temperature and pressure at the point where film thickness versus temperature was discontinuous. These critical values were used in an accepted semi-empirical equation [22] along with the simulation vapor density and pressure to produce a value for $\mathcal{L}$. This number was confirmed to be sufficiently accurate by measuring the condensation at the edge the molecular simulation away from the drop (averaged over $60 \sigma$ in $x$ ) and comparing it with thickness predictions based on this $\mathcal{L}$ value. This comparison is shown in figure 2. We see the slight accumulation of fluid because of the evaporation and condensation, and that our predicted value of $\mathcal{L}$ matches this. For comparison, predictions for both $\mathcal{L} / 2$ and $3 \mathcal{L} / 2$ fail to match the atomistic simulations. Though there is 


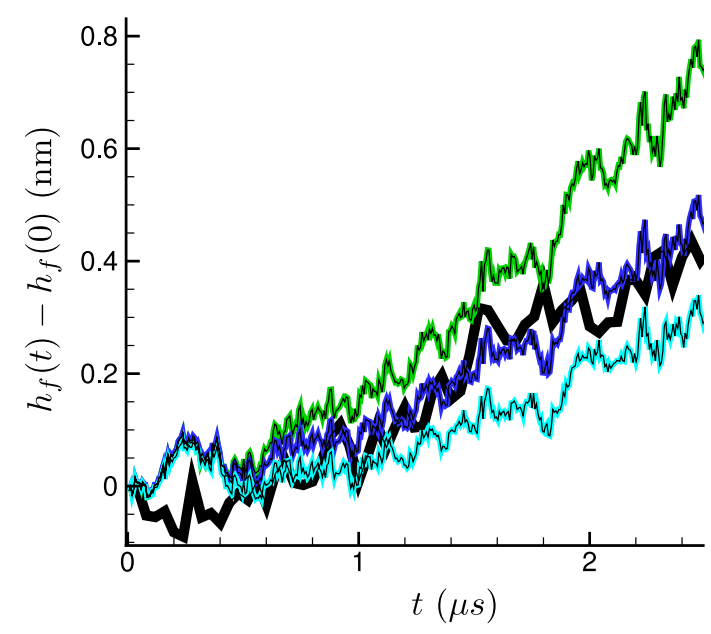

Figure 2. Precursor film thickness in black compared with predictions of (10) for different latent heats: $\mathcal{L} / 2, \mathcal{L}$, and $3 \mathcal{L} / 2$.

clearly finite evaporation and condensation, which motivated its inclusion in the model, it should also be clear evaporation played a relatively small role in the dynamics. The drop spread primarily by other mechanisms.

\section{Results and conclusions}

To quantify the spreading of the drop, we define its width $L$ via the second moment about its center $x_{\mathrm{c}}$ :

$$
L^{2}(t)=\frac{\int_{0}^{L_{b}} h(x, t)\left(x-x_{\mathrm{c}}\right)^{2} \mathrm{~d} x}{\int_{0}^{L_{b}} h(x, t) \mathrm{d} x},
$$

with $L_{b}$ the $x$ domain size. Figure 3 compares $L(t)$ for the atomistic simulation, the stochastically augmented lubrication equation, and the standard lubrication equation. Clearly, there is a poor comparison with the standard lubrication model, whereas the stochastic lubrication model appears to follow the atomistic simulation results.

Though this comparison is striking, it should be clear that this relative importance of the stochastic spreading term is not expected in all cases. Davidovitch et al [4] discusses why this type of thermal spreading would only be found in certain regimes. For self-similar spreading drops with initial height $h_{o}$, they showed that thermal forcing should dominate the surface tension only when

$$
h(t) \ll \frac{h_{o}^{2}}{x^{*}} \quad \text { and } \quad\left|x-x_{\mathrm{c}}\right| \gg x^{*},
$$

with $x^{*}=h_{o}^{7 / 6} W^{1 / 6} / l_{\mathrm{T}}^{1 / 3}$ and $h_{t}$ the current height of the drop. What (16) indicates is that drop needs to be relatively flat, so that the pressure supplied from the curvature in the interface is relatively small, and that stochastic stress dominated spreading will occur in regions farther away from the drop center than $x^{*}$. The first inequality is the more difficult to satisfy in atomistic simulations, because it requires relatively long run times, while the second inequality is often obtained in any large molecular simulation of spreading drops [10,9]. Clearly, the smallness

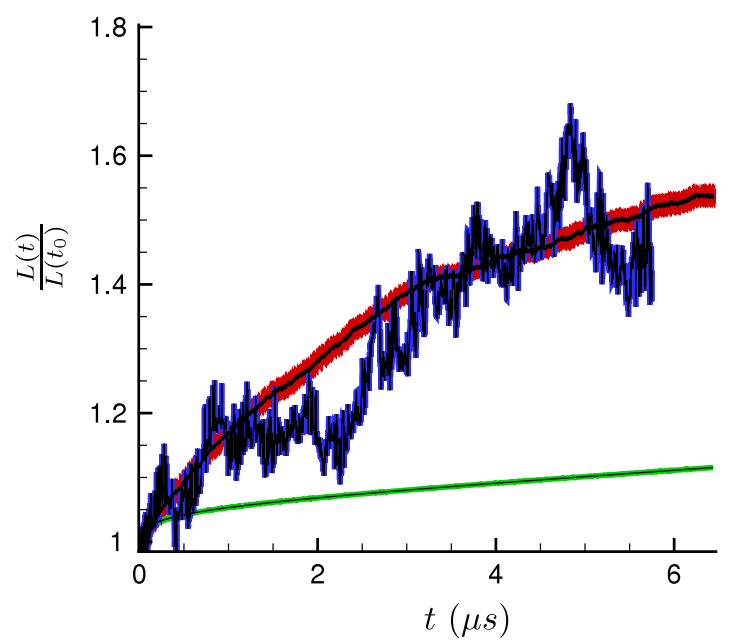

Figure 3. Drop spreading: molecular simulation, stochastic lubrication model (ensemble average of ten simulations), and the standard lubrication model.

of $W$ in our case makes both these conditions easier to satisfy. Our simulation ran almost $3 \times 10^{6} \sqrt{m \sigma^{2} / \varepsilon}$ but it still only slightly surpassed the first condition as a simple inequality, $h=$ $5.6 \mathrm{~nm}$ being only slightly less than $h_{o}^{2} / x^{*}=6.4 \mathrm{~nm}$. Other simulations of shorter spreading times or of higher viscosity drops would not spread far enough to enter into this regime. Another challenge is achieving enough spreading for it to be noticeable, which motivated our choice of fluid. The two-atompolymer model was selected as a balance between having a low enough viscosity that enough spreading could occur but small enough evaporation that spreading is still the dominant mode of fluid transport.

In conclusion, for the regime considered the thermal spreading of thin liquid films appears to more than double the spreading rate. It also appears that current stochastic fluid equations quantitatively reproduces this behavior.

\section{Acknowledgments}

This research was supported by an allocation of advanced computing resources supported by the National Science Foundation. The computations were performed on both Lonestar and Ranger at the Texas Advanced Computing Center. Funding from the National Science Foundation is also very gratefully acknowledged.

\section{References}

[1] Aarts D G A L, Schmidt M and Lekkerkerker H N W 2004 Direct visual observation of thermal capillary waves Science $304847-50$

[2] Ajaev V S, Homsy G M and Morris S J S 2002 Dynamic response of geometrically constrained vapor bubbles J. Colloid Interface Sci. 254 346-54

[3] Anderson H 1980 Molecular dynamics at constant pressure and/or temperature J. Chem. Phys. 72 2384-93

[4] Davidovitch B, Moro E and Stone H 2005 Spreading of viscous fluid drops on a solid substrate assisted by thermal fluctuations Phys. Rev. Lett. 95244505 
[5] Frenkel D and Smit B 1996 Understanding Molecular Simulation (San Diego, CA: Academic)

[6] Freund J B 2005 Atomic detail of an evaporating meniscus Phys. Fluids 17022104

[7] Grün G, Mecke K and Rauscher M 2006 Thin-film flow influenced by thermal noise J. Stat. Phys. 122 1261-91

[8] He G and Hadjiconstantinou N G 2003 A molecular view of Tanner's law: molecular dynamics simulations of droplet spreading J. Fluid Mech. 497 123-32

[9] Heine D R, Grest G S and Webb E B 2003 Spreading dynamics of polymer nanodroplets Phys. Rev. E 68061603

[10] Heine D R, Grest G S and Webb E B 2005 Surface wetting of liquid nanodroplets: droplet-size effects Phys. Rev. Lett. 95107081

[11] Hennequin Y, Aarts D G A L, van der Wiel J H, Wegdam G, Eggers J, Lekkerkerker H N W and Bonn D 2006 Drop formation by thermal fluctuations at an ultraloaw surface tension Phys. Rev. Lett. 97244502

[12] Hupert H E 1982 Flow and instability of viscous current down a slope Nature $\mathbf{3 0 0} 427-9$

[13] Jiang Z, Kim H, Jiao X, Lee H, Lee Y J, Byun Y, Song S, Eom D, Li C, Rafailovich M H, Lurio L B and Sinha S K 2007 Evidence for viscoelastic effects in surface capillary waves of molten polymer films Phys. Rev. Lett. 98227801

[14] Kim H, Jiang Z, Lee H, Lee Y J, Jiao X, Li C, Lurio L, Rafailovich M and Sinha S K 2007 Hydrodynamic surface fluctuations of polymer films by coherent $\mathrm{x}$-ray scattering Thin Solid Films $\mathbf{5 1 5} 5536-40$
[15] Kroger M, Loose W and Hess S 1993 Rheology and structural changes of polymer melts via non-equalibrium molecular dynamics J. Rheol. 37 1057-79

[16] Moseler M and Landman U 2000 Formation, stability, and breakup of nanojets Science 289 1165-9

[17] Rapaport D C 1997 Molecular Dynamics Simulation (Cambridge: Cambridge University Press)

[18] Schrage R W 1953 A Theoretical Study of Interface Mass Transfer (New York: Columbia University Press)

[19] Seo Y S, Koga T, Sokolov J, Rafailovich M H, Tolan M and Sinha S 2005 Deviations from liquidlike behavior in molten polymer films at interfaces Phys. Rev. Lett. 94157802

[20] Tanner L H 1979 Spreading of silicon oil drops on horizontal surfaces J. Phys. D: Appl. Phys. 12 1473-85

[21] Trokhymchuk A and Alejandre J 1999 Computer simulations of liquid/vapor interface in Lennard-Jones fluids: some questions and answers J. Chem. Phys. 111 8510-23

[22] Viswanath D S and Kuloor N R 1966 Heat of vaporization, surface tension, and temperature J. Chem. Eng. Data 11 69-72

[23] Wallace W E, Fisher D A, Efimenko K, Wu W and Genzer J 2001 Polymer chain relaxation: surface outpaces bulk Macromolecules 34 5081-2

[24] Wang J, Tolan M, Seeck O H, Sinha S K, Bahr O, Rafailovich M H and Sokolov J 1999 Surfaces of storngly confied polymer thin films studied by x-ray scattering Phys. Rev. Lett. 83 1-4 